\title{
El sismo del 16 de abril en Manabí visto desde la Ecología Política del desastre
}

\author{
The April's earthquake in Manabi \\ from the disaster Political Ecology
}

Elizabeth Bravo ${ }^{1}$

mbravov@ups.edu.ec

\section{Resumen}

Este trabajo hace una lectura del terremoto del 16 de abril 2016 desde la Ecología Política del desastre. Se plantea que, aun cuando todos los miembros de una comunidad pueden estar expuestos al mismo tipo de riesgo natural, hay una diferenciación en el grado de vulnerabilidad y marginalidad que enfrentan los distintos sectores. Se plantea además que hay un tipo de riesgo entre las élites, que ha sido facilitado por acción u omisión del Estado o de los gobiernos locales, como es el desarrollo turístico en la Ruta del Sol. Finalmente se propone una tipificación del nivel de vulnerabilidad enfrentado por los damnificados por el terremoto.

\section{Palabras claves}

Desastre, vulnerabilidad, riesgo, marginalidad, facilitación, shock.

\begin{abstract}
This work reads the earthquake of April 16, 2016, from the Political Ecology of the disasters. Although all members of a community could be exposed to the same type of natural risk, there is a differentiation in the degree of vulnerability and marginality that different sectors face. It is also suggested that there is a type of risk among elites, which has been facilitated by action or omission by the State or local governments, such as tourism development on the "Ruta del Sol". Finally, a classification of the level of vulnerability of the affected people by the earthquake, is proposed.
\end{abstract}

\section{Keywords}

Disaster, vulnerability, risk, marginality, facilitation, shock.

Forma sugerida de citar: $\quad$ Bravo, Elizabeth (2017). El sismo del 16 de abril en Manabí visto desde la Ecología Política del desastre. Universitas, XV(26), pp. 235-252.

1 Profesora e Investigadora de la Universidad Politécnica Salesiana (Grupo de Investigación en Ecología Política). Parte del Consejo Académico del Instituto de Estudios Ecologistas. Miembro de Acción Ecológica. 
El 16 de abril 2016, Ecuador vivió un sismo de magnitud 7.8, cuyo epicentro fue la población de Pedernales en la provincia de Manabí, dejando 670 fallecidos y miles de afectados. Las poblaciones más afectadas fueron Pedernales, Manta, Portoviejo, Canoa, Jama y Bahía de Caráquez en Manabí, y los cantones Muisne y Atacames en Esmeraldas.

Este no es el primer sismo de gran magnitud que se vive en las costas ecuatorianas. El Ecuador por encontrarse en el Cinturón de Fuego del Pacífico, es una zona geológicamente muy dinámica. Las costa ecuatoriana se encuentra en el margen de la zona de subducción de la placa oceánica de Nazca con las placas continentales de América del Sur y el Caribe, ambas separadas por el mega escudo Guayaquil- Caracas, lo que le hace muy vulnerable a terremotos y tsunamis de origen tectónico.

El 31 de enero de 1906, tuvo lugar un sismo de magnitud 8.8 con epicentro en la provincia de Esmeraldas y, minutos más tarde se generó un tsunami que arrasó numerosas poblaciones costeras de Ecuador y Colombia. Este fue un evento en la zona de subducción de la placa Nazca bajo la placa Sudamericana, en el Océano Pacífico.

Desde entonces, se han dado en la zona de Manabí y Esmeraldas (en una zona de unos $250 \mathrm{~km}$ ), otros cuatro terremotos con magnitud de 7 grados o más, desde inicios del siglo XX: 1942 (7.8 grados), 1958 (7.7 grados), 1979 (8.2 grados), 1998 (7.1 grados). Los epicentros de estos eventos están dentro de la zona de ruptura del mega evento de 1906 (Instituto Geofísico, 2011).

De acuerdo al Instituto Geofísico de la Escuela Politécnica Nacional, el terremoto de 1906:

Pese a estar considerado, junto con el de Chile de febrero de 2010, como el quinto terremoto más grande de la historia en cuanto a magnitud, el efecto sobre las casas que tuvo el sismo del 31 de enero de 1906 fue pequeño en relación con su gran magnitud. El factor determinante para ello fue el tipo de construcción que predominaba en la época: "Si todas estas casas hubieran estado hechas de ladrillos y piedras, toda la ciudad [de Tumaco] habría sido destruía por el primer impacto del movimiento, enterrando así bajo sus escombros a la mayoría de los habitantes, pero como estaban hechas de madera, muy dura además y muy bien encajada, poseen una gran elasticidad y resistieron muy bien a los movimientos" (Instituto Geofísico, 2011).

En este trabajo se quiere hacer una reflexión sobre los desastres naturales a la luz de la ecología política, abordando conceptos como riesgo, vul- 
nerabilidad, facilitación, marginalidad. Con estos elementos, se hace una tipificación de los efectos del terremoto del 16 de abril 2016 en Manabí y Esmeraldas.

\section{La Ecología Política de los desastres}

La Ecología Política estudia la interrelación de las sociedades humanas con la naturaleza. Analiza cómo se da el acceso, control y uso de los recursos naturales y del territorio, en una sociedad conformada por distintos grupos de poder. La Ecología Política surge como una crítica a la modernidad, en la medida que cuestiona los paradigmas existentes en torno al rol de los recursos naturales en los procesos económicos; y que mira a la naturaleza como un espacio de explotación, lo cual genera una serie de conflictos en torno al acceso de recursos que son disputados por actores con distintos grados de dotación de poder, como señala Alimonda:

Son las relaciones sociales de poder las que regulan el acceso, la disponibilidad y la utilización de los elementos de la naturaleza. Esta regulación realimenta al poder, y divide a las sociedades en función de esa disponibilidad y de su usufructo: de esa forma, las relaciones entre los humanos son intermediadas por la naturaleza, así como la naturaleza ha sido marcada por las acciones de los humanos, que han intervenido sobre ella consciente o inconscientemente. Es este el campo de reflexión que pretende la ecología política (Alimonda, 2008, p. 13).

La "ecología política del desastre" plantea varias preguntas sobre cómo opera tanto el poder como los aspectos geofísicos y ambientales que dan lugar a desastres y eventos catastróficos y los que se activan en el post-desastre. Esta sostiene que, en un escenario de desastre, se marginaliza a los más marginalizados, y se fortalecen a los que detectan el poder. La contribución de la ecología política del desastre es que logra combinar los aspectos políticos con los "naturales" en la interpretación de estos eventos (Claus et al., 2015).

Las decisiones que se toman frente a los desastres son profundamente políticas. Cuando estos desastres generan cambios ambientales o transforman las formas de producción no deseados, se ponen de manifiesto las relaciones de poder que existen en esos escenarios.

Existen algunos estudios sobre la relación entre los desastres naturales y las relaciones de poder que se dan, al momento de analizar los impactos 
de dicho desastre así como de interpretar las formas como se enfrenta la reconstrucción. Esta es materia de estudio del “Análisis de Vulnerabilidad”. El Análisis de Vulnerabilidad se usa para evaluar los elementos de exposición, susceptibilidad y resiliencia de cualquier sistema que está bajo amenaza, e identifica tres tipos de vulnerabilidad; 1) social, 2) económica, y 3) natural. En muchas ocasiones estos tres tipos de vulnerabilidad se sobreponen (Wisner et al., 2003).

Donner (2007) hizo un estudio sobre los efectos de los tornados en las regiones que están en zona de influencia de estos fenómenos naturales en Estados Unidos, entre los años 1998 a 2000. El investigador analizó la situación de la población rural, las minorías raciales y las personas que enfrentan tradicionalmente privación en la organización social. Los resultados revelan una fuerte correlación entre el tamaño y las rutas del tornado con el número de muertos y personas con lesiones, pero además, encontraron que son los factores demográficos y sociales los que desempeñan un papel central en la vulnerabilidad a los tornados.

La literatura demuestra que las personas con más bajos ingresos y poca educación, adultos mayores, mujeres, afroamericanos fueron los más afectados después del fenómeno Katrina en el Estado de Luisiana en Estados Unidos. Por ejemplo, se encontró que el $85 \%$ de los muertos eran mayores de 51 años, y más de la mitad eran mayores de 75 años (Batti y Das, 2016). Muchas de las personas que perdieron sus hogares eran afrodescendientes.

Pero además los desastres afectan a los ecosistemas, incrementado el nivel de daño. Por ejemplo, en el caso del huracán Katrina, se afectaron varios humedales y se eliminaron zonas de amortiguamiento de huracanes e inundaciones, exponiendo a varias poblaciones que antes estaban protegidas por estas zonas de amortiguamiento natural, a nuevos fenómenos climáticos como Katrina (Adeola y Picou, 2010; Picou y Hudson, 2010 y Balcia et al., 2012).

\section{Desastres naturales y acción humana}

Muchos desastres cuyo origen es considerado como "natural", tiene como origen, o está exacerbado por actividades humanas. Este es el caso de Katrina, el sexto huracán del Atlántico más intenso registrado, uno de los más destructivos y el que causó más víctimas mortales en Estados Unidos. 
Al menos 1833 personas fallecieron debido al propio huracán o las consiguientes inundaciones.

Aunque Katrina podría ser considerado como un desastre natural, la magnitud del huracán tuvo al menos dos causas no-naturales: a) el mayor número de muertes se registró en Nueva Orleans, que quedó inundada varias semanas porque un sistema de diques, que no había recibido el mantenimiento necesario, colapsándose muchos de ellos varias horas después de que el huracán hubiese continuado tierra adentro. b) tanto Katrina como otros huracanas en el Atlántico y el Pacífico se han multiplicado e incrementado su peligrosidad a causa del calentamiento global, relacionado con la quema de combustibles fósiles.

De igual manera, aunque el terremoto del 16 de abril 2016 ocurrió por causas puramente naturales, los impactos que éste genera y las evoluciones tanto en el ambiente como en la sociedad, tiene mucho más que ver con las relaciones entre actores sociales, antes que con las interacciones ecológicas de orden natural.

Ahora bien, ¿cuáles son las conexiones entre los riesgos que enfrentan las personas y las razones de su vulnerabilidad ante los peligros? ¿cómo se pueden percibir los desastres dentro de los patrones más amplios de la sociedad? ¿cómo analizarlos de manera que puede proporcionar una forma mucho más fructífera de construir políticas que puedan ayudar a reducir los desastres y a mitigar los peligros, mejorar las condiciones de vida y las oportunidades en general?

El punto crucial para entender por qué ocurren los desastres es que no son sólo los eventos naturales los que los causan. También son producto de entornos sociales, políticos y económicos (distintos del entorno natural), debido a la forma en que éstos estructuran la vida de diferentes grupos de personas, y aunque un desastre puede originarse por causas naturales, el que se convierta en una catástrofe depende de razones políticas, sociales y económicas.

Muchos aspectos del entorno social son fácilmente reconocibles: las personas viven en situaciones económicas adversas que les obligan a habitar regiones y lugares afectados por peligros naturales, ya sean las planicies de inundación de los ríos, las laderas de los volcanes o las zonas de terremotos. Sin embargo, hay muchos otros factores políticos y económicos menos obvios que subyacen al impacto de los peligros. Se trata de la manera en que los bienes, los ingresos y el acceso a otros recursos, como el conocimiento 
y la información, se distribuyen entre diferentes grupos sociales y diversas formas de discriminación que se producen en la asignación del bienestar y la protección social (Wisner et al., 2003).

Cuando ocurren eventos como un terremoto, estos se convierten en desastres cuando confluyen otros factores, que hacen que sea más difícil para la gente lidiar con ellos. Con frecuencia, las personas vulnerables sufren a menudo shocks que se refuerzan mutuamente, son repetidos, múltiples, a veces, simultáneamente, a sus familias, sus asentamientos y sus medios de subsistencia. Estos choques repetidos erosionan cualquier intento que se haya hecho para acumular recursos y ahorros. Los desastres frenan el desarrollo económico y humano a nivel de los hogares (cuando el ganado, los cultivos, los hogares y las herramientas son destruidos repetidamente) y en el nivel nacional cuando las carreteras, puentes, hospitales, escuelas y otras instalaciones resultan dañadas. El patrón de tales tensiones frecuentes, provocado por una amplia variedad de mecanismos de disparo "naturales", ha sido a menudo complicado por la acción humana, tanto por los esfuerzos para paliar los efectos del desastre como por la causalidad social de la vulnerabilidad (Wisner et al., 2003).

Por ejemplo, en Manabí hay un problema persistente de falta de agua, que se exacerbó con la ruptura de los sistemas de agua en los días posteriores al terremoto. De igual manera, esta es una zona que enfrenta variaciones climáticas muy erráticas, con períodos prolongados de sequías e inundaciones muy fuertes. En el cantón Rocafuerte, se había producido un poco antes del terremoto lluvias de tal intensidad, que se había desbordado la represas Poza Honda. El exceso de humedad en el suelo puso en mayor estado de vulnerabilidad a las viviendas, produciéndose muchas pérdidas. Igual cosa sucedió en zonas que habían sido muy afectadas por el fenómeno del Niño en el año 2010; así como en zonas donde el ecosistema del manglar (que es considerado como un zona de amortiguamiento frente a desastres naturales) había sido devastado por el avances de las camarones ${ }^{2}$.

La rápida urbanización aumenta el número de personas en riegos frente a un fenómeno natural, como lo demuestra el número de víctimas en el terremoto en Gujarat, India $(2001)^{3}$ y deslizamientos de tierra en Caracas, Ve-

2 Testimonio recogido a pobladores de Rocafuerte en diciembre 2016 y observación personal.

3 Más de 20 mil personas muertas y 167 mil heridos. 
nezuela en el año 19994. En el caso del terremoto en Ecuador, fue en Manta donde se reportó el mayor número de defunciones (219), a pesar de no ser el epicentro, pero es la ciudad con la mayor densidad poblacional de Manabí (1 046,34 habitantes por kilómetro cuadrado), y la novena a nivel nacional.

\section{Riesgo y vulnerabilidad}

Los seres humanos no son igualmente capaces de acceder a los recursos y oportunidades, ni están igualmente expuestos a los mismos peligros. El hecho de que las personas tengan o no suficiente tierra para cultivar, o acceso adecuado al agua, o un hogar decente, están determinados por factores sociales (incluyendo procesos económicos y políticos). Estos mismos procesos sociales también tienen un papel muy importante para determinar quién está más expuesto a riesgos y quién es más vulnerable: dónde viven y trabajan, y en qué tipo de edificios, su nivel de protección, preparación, información, riqueza y salud determinará su capacidad, no sólo de ser más o menos víctima de un desastre, pero también determina cómo afrontarlo. Así que la exposición de las personas al riesgo difiere según su clase, si son hombres o mujeres, cuál es su origen étnico, a qué grupo de edad pertenecen, si son discapacitados o no, estado de inmigración y en algunos países su religión (Wisner et al., 2003).

Para comprender los desastres no sólo debemos conocer los tipos de peligros que pueden afectar a las personas, sino también los diferentes niveles de vulnerabilidad y de riesgo entre diferentes grupos de personas, lo que está determinado por los sistemas sociales y el poder, y no sólo por las fuerzas naturales. Es necesario entender la exposición a un riesgo y la vulnerabilidad, en el contexto de los sistemas políticos y económicos que operan a escala nacional y local, pues es a ese nivel que se decide cómo varían los grupos de personas en relación con la salud, los ingresos, la seguridad del edificio, la ubicación del trabajo y del hogar, y así sucesivamente.

Se define como riesgo la posibilidad de que se produzca un evento en el que alguien sufre perjuicios o daño, multiplicado por la magnitud del evento. En la zona de Manabí/ Esmeraldas ha habido cuatro sismos impor-

4 Conocida como Tragedia de Vargas, constituye el desastre con el mayor número de víctimas mortales por un alud de barro. 
tantes en el último siglo, por lo que toda la población que habita esta zona está bajo condiciones de riesgo, pero hay una distinción en cómo los diferentes segmentos de la sociedad pueden enfrentarlo. Ahí entra el concepto de vulnerabilidad.

Para Wisner et al. (2003), la vulnerabilidad está determinada por las características de una persona o grupo y su situación que influyen en su capacidad de anticipar, afrontar, resistir y recuperarse del impacto de un evento o proceso natural extremo. Se trata de una combinación de factores que determinan el grado en que la vida, el sustento, la cultura, la propiedad y otros bienes de una persona, se ponen en riesgo por un evento discreto e identificable (o una serie o cascada de tales eventos) en la naturaleza y en la sociedad. La vulnerabilidad implica por tanto, magnitudes variables, con personas o comunidades con un mayor grado de vulnerabilidad que otros.

Un desastre puede ser abordado desde el punto de vista del riesgo, que es una visión más tecnocrática y que se centra en el evento como tal; o desde la vulnerabilidad, que se centra en la gente o comunidades víctimas del desastre. Para Sarewitz et al. (2003), mientras la reducción de la vulnerabilidad es un derecho humano, la reducción del riesgo no lo es.

\section{Marginalidad y facilitación}

En su estudio sobre el incendio de Rodeo-Chediski en las Montañas Blancas de Arizona que tuvo lugar en 2002 y que destruyeron más de 80000 hectáreas, Collins (2008) nos introduce a dos conceptos: marginalidad y facilitación.

Para Susman et al. (1984), es marginal quien es forzado a salir de su tierra a zonas muy pobres o insuficientes; o aquellos que viven en zonas peligrosas o insalubres. El concepto de marginalidad, que es central en los estudios de ecología política, fue desarrollado para explicar cómo los grupos económicos con menor dotación de poder, están expuestos a condiciones de vulnerabilidad a cambios socio-ambientales (Collins, 2008).

Si bien la "marginalidad" se produce cuando ciertas poblaciones son empujadas a zonas peligrosas (Hewitt, 1997), Collins señala que el estudio de la marginalidad puede ser consolidado a través de un enfoque en el concepto de "facilitación", que sugiere como a ciertos grupos privilegiados se les proporciona ayudas para acceder a espacios peligrosos, pero que están protegidos por seguros privados o por políticas públicas. Un ejemplo cla- 
ro es la ampliación urbana en las faldas del Pichincha por parte de élites de Quito, donde barrios amurallados coexisten con barrios marginales.

En su estudio sobre los incendios en Arizona Collins (2008) señala que esta es una zona que en el pasado estuvo dedicada a la ganadería y extracción maderera pero en las últimas décadas se convirtió en una zona residencial. Los bosques se hicieron muy espesos, y los incendios empezaron a proliferar. Los impactos del incendio fueron diferenciados si se trataba de zonas de desarrollo urbano de élite, con casa de fin de semana y campos de golf. En este caso, los daños causados por el incendio estuvieron cubiertos por seguros, lo que no pasó en las zonas marginales de población indígena y latina. Este es un ejemplo que muestra que, aun cuando todas las comunidades asentadas en las Montañas Blancas de Arizona sufrieron los efectos del incendio, la forma como se trató la problemática fue diferencial.

La marginalización y facilitación pueden generar diferentes tipos de riesgos, que reflejan las desigualdades sociales. Las clases privilegiadas están utilizando y controlado cada vez más recursos para exteriorizar riesgos y capitalizar en oportunidades ambientales. Los conceptos de marginalidad y facilitación no son excluyentes, pero generan distintos niveles de vulnerabilidad.

Este es un marco que puede ayudar a entender el terremoto del 16 de abril 2016. El sismo golpeó a pobres y ricos por igual, porque lo que determinó los epicentros fue la geología y no la acción humana... pero, a pesar de la historia geológica de la zona, ya descrita; a lo largo de lo que se llama "la Ruta del Sol", se llevó a cabo un gran desarrollo turístico, desechando las viejas estructuras de caña (Saltos, 2016, p. 273).

No se cumplió ninguna norma de construcción, y los gobiernos locales se limitaron en proveer servicios básicos y el Gobierno central a abrir carreteras para promover el turismo, en esa zona de tiene historia sísmica reciente.

Por eso las pérdidas para el sector turísticos fueron enormes: 64,5 millones de dólares; el 19\% de los edificios turísticos colapsaron, el 33\% fue parcialmente afectado y el $48 \%$ tuvo daños menores (El Universo, 29 de julio 2016).

Al sur de Manta, zona que no fue esta vez afectada por el sismo, hay una dinámica de expansión del sector inmobiliario, donde las ciudadelas de lujo se abren entre los otrora pueblos de pescadores artesanales. Esta es una tendencia que se mantiene aún después del terremoto (El Comercio, 23 de mayo 2016). 
Pero todas estas edificaciones estaban aseguradas, por lo que, aunque toda la población que vive en esta zona está en peligro, algunos viven en estado de marginalidad, otros de vulnerabilidad y otros se benefician de lo que Collins llama la "facilitación".

\section{La doctrina del shock}

La doctrina del shock o el "auge del capitalismo del desastre" es propuesto por Naomi Klein quien demuestra a través de varios ejemplos cómo, bajo condiciones de desastre (tsunami en Sri Lanka, las inundaciones de Katrina), se toman decisiones y se implementaron políticas públicas que no son posibles sin un escenario de emergencia, y que en muchos casos favorecen a los grupos de poder.

Una primera experiencia de la doctrina del shock se remonta al año del Niño 1877-1878, que produjo grandes inundaciones en esta parte del Pacífico, pero que en otras regiones generó sequías generalizadas. A propósito de este evento, Davis (2001, pp. 267-269) reporta cómo el imperio británico desmanteló un sistema altamente eficiente para responder a las crisis climáticas en sus colonias asiáticas, lo que produjo impactos negativos en esa región.

Posteriormente, en la década de 1930 en Estados Unidos, la "gran depresión" coincidió con un "fenómeno natural" que duró 8 años (entre 19311939), caracterizado por fuertes tormentas de arena'; grandes nubes de polvo y arena tan espesas que escondían el sol, dando lugar a "ventiscas negras". Este fenómeno se manifestó en la zona llamada "Grandes Llanuras", lo que obligó a los colonos europeos que habían llegado a estas tierras unas décadas atrás, a migrar hacia California, desapareciendo una forma de producción basada en la agricultura familiar, creándose los grandes latifundios que aún imperan en esa región, pues los granjeros no pudieron pagar las deudas contraídas como parte de las políticas públicas desarrolladas durante la ocupación de las grandes llanuras (Holleman, 2016).

En tiempos más modernos, Klein describe cómo el tsunami que azotó las playas del sur y sureste asiático el 26 de diciembre 2004, que dejó sin vida a 250 mil personas y 2,5 millones se quedaron sin hogar en la región, sirvió

5 Dust bowl (en inglés), que afectó a 400000 kilómetros cuadrados. 
de escenario para poder concretar un ambicionado plan turístico en la zona de Arugam (Sri Lanka). Después de desalojar a la población local con el argumento de esa era una zona de riesgo de tsunami, se empezó un programa de reconstrucción de desarrollo turístico. La gente fue llevada a campamentos temporales, que con el tiempo se consolidaron en tugurios (Klein, 2007).

A consecuencia del mismo tsunami, en las Maldivas, varias islas fueron desalojadas por ser "inseguras e inadecuadas para ser habitadas", y se lanzó un programa agresivo de traslado de poblaciones de pescadores a islas "más grandes y seguras", dejando 35 islas disponibles para el turismo.

El tema del uso de los desastres para militarizar zonas, y promover su privatización es ampliamente desarrollado por Klein (2007), en relación al desastre de Katrina. Este desastre sirvió además para privatizar los colegios públicos (de 123 escuelas públicas se pasó a 4); el desmantelamiento del sindicato de maestro; el vaciamiento de barrios de población afro, entre otros.

Podrían identificarse al menos dos indicios de la doctrina del shock en el terremoto del 16 de abril 2016 en la costa ecuatoriana:

a) La imposición de medidas de seguridad y control adicional sobre la población. Los decretos de estado de excepción decretados desde que tuvo lugar el terremoto, estuvieron a cargo del Ministerio de Coordinación de Seguridad y de las Fuerzas Armadas. Aunque la declaración de estado de excepción se planteaba "enfrentar, recuperar y mejorar las condiciones adversas del sismo", no fueron los ministerios de Salud o Inclusión Sociales los que estuvieron a cargo de implementar esos objetivos.

Las zona cero de Manta, Portoviejo y Pedernales y los albergues estatales fueron militarizados. A través de los estados de excepción, se suspendió el derecho a la inviolabilidad de domicilio y libre circulación de los afectados por el terremoto. En las horas siguientes al terremoto, no se le permitió a la gente entrar en sus casas para recuperar sus pertenencias.

b) Lo descrito por Klein en el caso de Sri Lanka y Las Maldivias nos recuerda mucho a lo que está sucediendo en la Isla de Muisne. El 21 de junio 2016 a través de una resolución se declara a toda la isla como una zona de riesgo, porque podría ser sujeta de inundaciones en caso de un tsunami, y que por lo tanto, se prohíbe los asentamientos humanos en la isla (Secretaría de Riesgo, 2016).

Lo que llama la atención es que el Ministerio de Turismo (2016) publica el 6 de junio 2016, el proyecto "Programa Nacional de Destinos Turísticos 
de Excelencia" donde se incluye "Intervención al Destino Complementario Muisne".

\section{Tipificación de los afectados por el terremoto desde el punto de vista de vulnerabilidad}

Aunque un desastre como el terremoto del 16 de abril afectó a todos quienes estuvieron en su zona de influencia, la respuesta frente a los mismos, fueron distintas en los diferentes segmentos de la sociedad, de acuerdo al grado de vulnerabilidad de cada uno, como suele ocurrir en estos casos (Johnston, 1997; Willow, 2014).

Manabí es una provincia de grandes desigualdades. Coexisten los grandes empresarios atuneros, con un $76 \%$ de la población con necesidades básicas insatisfechas. Hay muchas poblaciones que aún antes del terremoto no tenían acceso al agua potable, y que para proveerse del líquido vital tenían que comprar de tanqueros.

A raíz del terremoto, unas 70 mil personas se quedaron sin hogar (El País, 16 de mayo, 2016), y analizar el destino de ellas permite hacer una tipificación de la vulnerabilidad frente al desastre ${ }^{6}$.

\section{a) Por el tipo de respuesta}

i. Asentamientos urbanos: Los grupos más vulnerables son aquellos perdieron su casa en las zonas urbanas, y que por diversas razones no acudieron a los albergues oficiales, y no forman parte de un tejido social que pueda darles acogida. Ellos se quedaron a vivir en refugios improvisados, donde el agua de lluvia se acumula e infiltra en la zona de vivienda, no tienen acceso a servicios básicos, lo que genera graves problemas sanitarios (La Nación, 2 de mayo 2016). Son personas muy marginales: en algunos casos no tienen cédula de identidad y los niños no tienen partida de nacimiento. Hay mujeres solas con 4 o 6 hijos a su cargo. Se dan casos de consumo de drogas y alcohol. Varias familias son recicladoras de basura. En estos grupos se generan liderazgos improvisados que controlan el destino de

6 Esta tipificación se hizo luego de varios recorridos y entrevistas realizadas en las zonas del desastre, sobre todo en Manabí. 
quienes llegan a estos lugares, como se pudo evidenciar en una visita de campo a un refugio en Pedernales.

ii. Asentamientos rurales: Quienes perdieron sus casas en zonas rurales, pero que no tenían títulos de propiedad de su terreno. Estas familias no se movieron a ningún albergue, sino que se refugiaron en espacios improvisados, con frecuencia a un lado de su casa destruida. Hasta diciembre 2016 se veían casas destruidas con carpas de plásticos o en el mejor de los casos, carpas donadas por el Programa de las Naciones Unidas para los Refugiados u otras agencias de ayuda. Ellos no acudieron a los albergues estatales por miedo a perder su tierra.

iii. Albergados oficiales: Luego está el grupo que perdió su casa y que acudió a los albergues del Estado. Son familias que no tenían ninguna propiedad, y tienen la esperanza que se les dé una casa nueva en algunos de los reasentamientos. Inmediatamente después del sismo, se abrieron 32 albergues oficiales, en donde se alojaron unas 33 mil personas. A inicios de marzo 2017, se mantenían albergados 1029 familias en siete cantones en las provincias de Esmeraldas y Manabí. La mayor parte está en Muisne con 431 familias y Pedernales con 255 (El Universo, 9 de marzo, 2017). Entre los albergados hubieron 268 personas con discapacidad, un grupo aún más vulnerable.

iv. Beneficiarios de bono de acogida: Las familias que vivían en un entorno familiar más cohesionado optaron por el bono de acogida, que es un subsidio que se entrega a quienes reciban a sus familias o vecinos. Ellos estuvieron en mejores condiciones que los grupos anteriores, porque pueden desenvolverse en un ambiente familiar. El bono tuvo una duración de tres meses.

v. Beneficiarios de bono de alquiler: Otras familias recibieron un bono de alquiler, pero el problema es que en algunos poblados, como Pedernales, donde la destrucción de las edificaciones fue muy grande, los lugares de arriendo escasearon. En el caso de familias que perdieron todas sus pertenencias esta tampoco fue una buena opción. Este bono fue por seis meses.

vi. Beneficiarios de bono de reconstrucción: Hay otro grupo de familias cuyas casas fueron afectadas, pero no destruidas. El Estado proveyó a estas familias bonos de construcción, reconstrucción y repara- 
ción ${ }^{7}$. No se beneficiaron de este bono quienes no tenían títulos de propiedad, es decir, los más marginales.

vii. Beneficiarios de casas nuevas: El Estado construyó casas en zonas urbanizadas o terrenos propios para algunas familias ${ }^{8}$. En un viaje técnico hecho en la zona rural de Manabí en diciembre 2016 se constató una gran diversidad de diseños de casas: mientras que en zonas de inundación, algunas casas nuevas estaban soportadas por pilotes, otras estaban construidas a ras del suelo, lo que aumenta la vulnerabilidad frente al desbordamiento de ríos.

viii.Beneficiarios de agencias de cooperación: Algunas agencias de cooperación y organizaciones no gubernamentales construyeron casas o albergues temporales a familias que estaban organizadas. La organización social en Manabí ha sido muy débil en las últimas décadas, por lo que el grado de vulnerabilidad en la región aumenta, por otro lado podemos apreciar cómo la organización empodera a sus miembros.

ix. Beneficiarios de la facilitación: Las personas con mayores recursos económicos, los empresarios hoteleros y otros grupos empresariales, como se ha visto antes, habían asegurado sus inmuebles por lo que cualquier pérdida que tuvieron, les fue restituida. De hecho, las aseguradoras pagaron un total de 374 millones de dólares; recibieron 38423 reclamos, de los cuales, en enero del 2017 el 87\% habían sido ya indemnizados.

x. Los que buscan el control de sus vidas: son el grupo de personas que se resisten al desalojo, la desterritorialización o a depender de la ayuda oficial, y que al contrario, están desarrollando alternativas sustentables, ya sea por sus propios esfuerzos, o con apoyo externo.

xi. Víctimas de la doctrina del shock: a quienes se les aplicó medidas de desalojo, aduciendo que viven en una zona de riesgo -lo que posiblemente es verdad-, pero en cuyo territorio se está gestando procesos de facilitación para el desarrollo turístico.

7 Quien reciba el bono de reparación paga 400 dólares en 16 meses. El resto es subsidiado por el Estado.

8 Para viviendas nuevas el bono es de diez mil dólares + IVA, y para reparación de cuatro mil dólares. El damnificado tiene que hacer un copago del 10\%: para casa nueva el damnificado debe pagar mil dólares en 36 cuotas de 27,77 cada una. 


\section{Discusión}

En la Ecología Política el riesgo, es la combinación de la exposición a peligros biofísicos y la capacidad de una persona o comunidad para anticiparse, responder y recuperarse del evento peligroso. La vulnerabilidad se relaciona inevitablemente con el poder, con la capacidad de ejercer el control sobre un evento. La vulnerabilidad por otro lado, es la combinación de factores que influyen en el grado en que la vida, la propiedad o los bienes se ponen en riesgo por la ocurrencia de un evento de peligro. Tales factores dependen del contexto, pero a menudo están influidos por el género, la clase, la edad, la raza o el origen étnico (Wisner et al., 2004).

En el análisis sobre el terremoto de Manabí podemos ver que todos los grupos estuvieron afectados, desde el punto de vista biofísico al mismo fenómeno (vulnerabilidad natural), aunque el riesgo aumentó porque a ciertos grupos de poder (inmobiliarias, sector turístico), se le facilitó por acción u omisión, la construcción de edificaciones inadecuadas en términos estructurales, el uso de materiales no aptos y el desarrollo de infraestructura pesada en zonas de riesgo.

Por otro lado, podemos ver que, lo que varió fue la respuesta y la capacidad de controlar su vida y su subsistencia después del desastre de los distintos grupos. Los sectores económicamente más favorecidos, pudieron cobrar el seguro por las pérdidas sufridas, y en algunos casos, inclusive mejoraron sus propiedades, como se pudo evidenciar en testimonios recogidos en Manabí por la autora después del terremoto.

En otros casos, las personas que se albergaron en las instalaciones estatales han perdido control de sus vidas porque muchas de ellas han pasado casi un año en espera de que venga la ayuda estatal, la que puede llegar o no, lo que constituye también una forma de vulnerabilidad. La recuperación del tejido social de este grupo va a ser muy dura. Es posible que este grupo esté en peores condiciones que quienes están refugiados a un lado de su casa, porque por lo menos no ha perdido control sobre su vida y territorio (vulnerabilidad social).

Se ha visto también el poder de la organización, pues sobre todo en el medio rural, se puede apreciar que las familias y comunidades que pertenecen a algún espacio organizacional han podido, no sólo acceder a una ayuda de mejora calidad, sino que también han iniciado procesos de reconstrucción integral. Este es el caso de quienes han decido quedarse en la Isla de 
Muisne a pesar del decreto de desalojo, y otras comunidades en Manabí y Esmeraldas.

La vulnerabilidad depende también de modalidades de ocupación territorial, y en este caso hemos visto el cambio en el uso del territorio: de comunidades de pescadores artesanales, poco densas desde el punto de vista poblacional, y con construcciones ligeras, más aptas para enfrentar un sismo como el que tuvo lugar en 2016; se pasó a un desarrollo inmobiliario y turístico que tenía visos de seguir expandiéndose, donde no se tomó en consideración que estaba asentado en una región sísmica, a pesar de la historia geológica del siglo XX, donde ocurrieron tres sismos de gran magnitud en la región.

Finalmente podemos ver cómo ha operado la Doctrina del Shock propuesta por Klein, en especial cuando se quiere usar el sismo para convertir a la isla de Muisne en un centro turístico, e implementar cambios en el uso del territorio.

Finalmente, es importante señalar que se ha sugerido que la reducción de la vulnerabilidad debe ser enmarcada en el ámbito de derechos humanos fundamentales, que la sociedad moderna tiene la obligación de asegurar que todos las personas y comunidades vivan con un nivel básico de protección contra la amenaza de desastres (Sarewitz et al., 2003), especialmente en países como el Ecuador que está amenazado por múltiples factores de riesgo, como son las erupciones volcánicas, sismos, fenómeno de El Niño, cambio climático...

Agradecimientos: A la Universidad Politécnica Salesiana que financió esta investigación, y a Viviana Pino, Verónica Freire, Adriana Ortiz, Janeth Martínez y Mauricio Pino por el apoyo en el trabajo de campo.

\section{Bibliografía}

Adeola, F.O., \& Picou, J.S. (2010). Race, social capital and the health impacts of Katrina: Evidence from Louisiana and Mississippi Gulf Coast. Hum Eco. Rev. 19: 10-24. doi: 10.3390/ijerph13020239.

Alimonda, H. (2008). Introducción. En: H. Alimonda (Coord.), Gestión ambiental y conflicto social en América Latina (pp. 13-21) Buenos Aires: CLACSO.

Claus, A. et al. (2015). Disaster, degradation, dystopia. En: Raymond Bryant (Ed.), The International Handbook of Political Ecology (pp. 291- 303). Cheltenham: Edward Elgar Publishing, Inc. 
Collins, T. (2008). The political ecology of hazard vulnerability: marginalization, facilitation and the production of differential risk to urban wildfires in Arizona's White Mountains. Journal of Political Ecology, 15, 21-43.

Davis, M. (2001). Late Victorian Holocausts. El Niño Famines and the Making of the Third World. Londres: Verso.

Donner, W.R. (2007). The political ecology of disaster: an analysis of factors influencing U.S. tornado fatalities and injuries, 1998-2000. Demography, 44 (3), 669-85. doi:10.1353/dem.2007.0024

El Comercio, 23 de mayo (2016). Santa Marianita es el núcleo inmobiliario de Manta. http://www.elcomercio.com/tendencias/santa-mariana-nuevo-nucleoinmobiliario.html

El Universo 9 de marzo (2017). Hoy se cierran albergues de Manta y Portoviejo. http://www.eluniverso.com/noticias/2017/03/09/nota/6080122/hoy-secierran-albergues-manta-portoviejo

El Universo, 29 de julio (2016). En Turismo se estima pérdidas por $\$ 64,5$ millones tras terremoto en Ecuador. http://www.eluniverso.com/noticias/2016/07/29/ nota/5714019/turismo-se-estiman-perdidas-645-millones

Hewitt, K. (1997). Region of Risk: A geographical Introduction to Disasters. Essex: Longman.

Holleman H. (2016). De-naturalizing ecological disaster: colonialism, racism and the global Dust Bowl of the 1930s. Journal of Peasant Studies, 44(1), 234-260.

Instituto Geofísico (2011). A 105 años del terremoto y tsunami de Esmeraldas, ¿Está Ecuador preparado para un evento similar. Escuela Politécnica Nacional. http://www.igepn.edu.ec/servicios/noticias/344-a-105-a\%C3\%B1osdel-terremoto-y-tsunami-de-esmeraldas-\%C2\%BFest $\% \mathrm{C} 3 \% \mathrm{~A} 1$-ecuador-preparado-para-otro-evento-as $\% \mathrm{C} 3 \% \mathrm{AD}$ ?

Klein, N. (2007). La doctrina del shock: El auge del capitalismo del desastre. Buenos Aires: Paidos.

Ministerio de Turismo (2016). Proyecto: K001 Mintur - Programa Nacional de Destinos Turísticos de Excelencia. Ficha informativa de proyecto 2016. Viceministerio de Gestión Turística Subsecretaría de Destinos. Dirección de Proyectos Especiales. http://www.turismo.gob.ec/wp-content/ uploads/2016/06/Proyecto-Destino-Turisticos-de-Excelencia.pdf

Noticias 24, 2 de diciembre (2010). El 80\% de la Guajira venezolana se encuentra inundada. http://www.noticias24.com/actualidad/noticia/183416/el80-de-la-guajira-venezolana-se-encuentra-inundada/ 
La Nación, 2 de mayo (2016). Tras el sismo, las lluvias hacen estragos en Ecuador y temen un brote de zika. http://www.lanacion.com.ar/1894682-ecuadorsismo-zika-lluvias-brote

Picou, J.S., \& Hudson, K. (2010). Hurricane Katrine and mental health: a Research note on Mississippi Gulf Coast resident. Social Inq., 80, 513-524. DOI: 10.1111/j.1475-682X.2010.00345.x

Saltos, N. (2016). Nuda vida, biopoder y solidaridad. Las políticas del desastre. Revista Andaluza de Antropología, 11, 270-287.

Sarewitz, D., Pielke, R., \& Keykhah, M. (2003). Vulnerability and Risk: Some Thoughts from a Political and Policy Perspective. Risk Analysis, 23 (4), 805-810. DOI: 10.1111/1539-6924.00357

Secretaría de Gestión de Riegos (2016). Resolución No. SGR-073-216 del 22 de junio 2016.

Susman, P., O’Keefe, P., \& Wisner, B. (1984). Global disasters: a radical interpretation. En: K. Hewitt (Ed.), Interpretation of calamity (pp. 264-283). Boston: Allen \& Urwin.

Willow, A. (2014). The new political of environmental degradation: un/expected landscapes of disempowerment and vulnerability. Journal of Political Ecology, 21, 238-257.

Fecha de recepción: 6/04/2017; fecha de aceptación: 23/06/2017;

fecha de publicación: 30/06/2017 